\title{
Efeitos do aumento da demanda inspiratória na estabilidade postural de idosos
}

Effects of increased inspiratory demand on postural stability in the elderly

Karina Pitombeira Pereira Pedro1; Thalles Andrade Marques Pereira1; Mauro Gonçalves1

1 Universidade Estadual de São Paulo -UNESP (Júlio de Mesquita Filho), Brasil.

Detalles del artículo:

Número de palabras: 2.496; Tablas: 2; Figuras: 1; Referencias: 13

Recibido: febrero 2020; Aceptado: abril 2020; Publicado: septiembre 2020

Conflicto de interés: El autor declara que no existen conflictos de interés.

Correspondencia del autor: Karina Pitombeira Pereira Pedro, ker.manutencao@gmail.com

\section{Resumo}

Introduction: Introdução: $\mathrm{O}$ envelhecimento pode provocar alterações fisiológicas e funcionais, e essas por sua vez, podem acarretar no declínio das funções e da capacidade funcional, e assim gerar um comprometimento na autonomia e a mobilidade, e aumentar o risco de quedas. $\mathrm{O}$ envelhecimento também está associado a alterações biológicas que comprometem a função muscular inspiratória. Existem estudos que mostraram que toda musculatura do tronco trabalha conjuntamente com a função de proteger a coluna e preservar o controle postural, incluindo os músculos inspiratórios, porém, o papel do diafragma na respiração sempre toma precedência sobre a função postural. Objetivo: Verificar a influência do incremento na demanda inspiratória sobre a estabilidade postural de idosos, por meio de um estudo de casos. Métodos: Três idosos, realizaram inicialmente a avaliação da pressão inspiratória máxima (PImáx), e em seguida foram submetidos a um teste de estabilidade postural. No equipamento Biodex Balance System SD, realizaram o protocolo do próprio dispositivo denominado 'Postural Stability Test' (PST), com o intuito de avaliar o índice de instabilidade em duas condições distintas. Na primeira condição os idosos realizaram o teste e podiam respirar normalmente, sem realizar esforços e tentando se manter equilibrado sobre a plataforma do equipamento; já na segunda condição os idosos foram avaliados realizando a respiração através do equipamento Power Breathe ${ }^{\circledR}$ e usando um clipe nasal. Para realização dessa condição de aumento na demanda inspiratória foi adotada uma sobrecarga de $50 \%$ do valor da PImáx, avaliada no início. Resultados: Nas oscilações anterior-posterior todos os idosos apresentaram maior índice de instabilidade no teste com aumento da demanda inspiratória e para as oscilações direita-esquerda dois dos idosos avaliados apresentaram maior índice de instabilidade nessa condição. Conclusão: $\mathrm{O}$ aumento na demanda inspiratória fornecido pelo dispositivo Power Breathe ${ }^{\circledR}$ foi capaz de alterar a equilíbrio de idosos, aumentando a instabilidade postural dos avaliados. Consideramos que para resultados mais esclarecedores seja necessário a realização de um estudo randomizado e controlado com uma amostra maior. 
Palavras-chave: músculos inspiratórios, "Biodex Balance System”, idosos.

Abstract

Introduction: Aging can cause physiological and functional changes, and these, in turn, can lead to a decline in functions and functional capacity, thus generating a compromise in autonomy and mobility, and increasing the risk of falls. Aging is also associated with biological changes that compromise inspiratory muscle function. There are studies that have shown that all trunk muscles work together with the function of protecting the spine and preserving postural control, including inspiratory muscles, however, the diaphragm's role in breathing always takes precedence over postural function. Objective: To verify the influence of the increase in inspiratory demand on postural stability in the elderly, through a case study. Methods: Three elderly people initially performed the maximal inspiratory pressure (MIP) assessment, and then underwent a postural stability test. In the Biodex Balance System SD, they performed the protocol of the device called 'Postural Stability Test' (PST), in order to assess the instability index under two different conditions. In the first condition, the elderly performed the test and could breathe normally, without making efforts and trying to stay balanced on the equipment platform; in the second condition, the elderly were evaluated performing breathing using the Power Breathe $®$ equipment and using a nasal clip. To achieve this condition of increased inspiratory demand, an overload of $50 \%$ of the MIP value was adopted, assessed at the beginning. Results: In the anterior-posterior oscillations, all the elderly individuals presented a higher rate of instability in the test with an increase in inspiratory demand and for the right-left oscillations, two of the evaluated elderly individuals presented a higher rate of instability in this condition. Conclusion: The increase in inspiratory demand provided by the Power Breathe ${ }^{\circledR}$ device was able to alter the balance of the elderly, increasing the postural instability of the individuals evaluated. We consider that for more clarifying results it is necessary to carry out a randomized controlled study with a larger sample.

Key words: inspiratory muscles, Biodex Balance System, elderly.

\section{INTRODUÇÃO}

Estudos apontam que o envelhecimento populacional é um processo mundial que pode ocasionar alterações em diversos sistemas, e especialmente nas estruturas e funções musculoesqueléticas (Santos Andrade \& Bueno, 2009; Caserotti, 2010). Estima-se que no Brasil, existem atualmente 21,7 milhões de idosos, representando mais de $10 \%$ da população. As projeções indicam que no ano de 2040, o Brasil terá 55 milhões de idosos, e estes representarão $27 \%$ da população. (IBGE, 2020).

O envelhecimento pode provocar também alterações fisiológicas e funcionais que podem acarretar no declínio das funções e da capacidade funcional, e assim gerar um comprometimento na autonomia e a mobilidade, e aumentar o risco de quedas (Studenski et al.,2011). Também está associado a alterações biológicas que comprometem a função muscular inspiratória (Britto et al. 2009). Em particular, a força dos músculos inspiratórios diminuiu gradualmente a partir dos 65 anos de idade (Enright et al. 1994). Esses declínios na função respiratória relacionados à idade podem alterar direta e indiretamente a contribuição dos músculos inspiratórios para o equilíbrio. Em evidências recentes também foi sugerido que a fraqueza muscular inspiratória pode contribuir nos déficits para se equilibrar durante as atividades diárias (Janssens et al. 2014). 
Foi sugerido que o treinamento muscular do tronco (isto é, treinamento de força abdominal e treinamento de exercícios de Pilates) pode melhorar o equilíbrio e, portanto, ser usado como uma intervenção de prevenção de quedas para adultos mais velhos (Granacher et al. 2013). No entanto, a contribuição dos músculos do tronco para o equilíbrio ainda não é totalmente clara.

Toda musculatura do tronco trabalha conjuntamente com a função de proteger a coluna e preservar o controle postural, e se contraem de forma automática em antecipação às ações que desestabilizam ou sobrecarregam a estabilidade postural. As contrações do diafragma ocorrem da mesma forma, mas o papel do diafragma na respiração sempre toma precedência sobre a função postural (Hodges, et al., 2002). Outros estudos apontam que a ação estabilizadora do músculo diafragma funciona de maneira indireta, aumentando a pressão intra-abdominal para apoiar a coluna, e diretamente, por co-contração contínua, contribuindo para a estabilização postural (Hodges et al., 2005; Hodges \& Gandevia, 2000).

Portanto, isso levanta a questão de saber o quanto os músculos inspiratórios, em especial o diafragma, podem influenciar no equilíbrio de idosos. Torna-se curioso saber se um aumento na demanda sobre o sistema muscular inspiratório usando um dispositivo de limiar de pressão, pode interferir na função de equilíbrio por parte da musculatura inspiratória, diminuindo ainda mais a capacidade de equilíbrio em idosos.

Neste sentido, entendemos que uma forma de analisar o equilíbrio e contribuir em pesquisas que possam inovar na prevenção de quedas em idosos seria a medição de parâmetros biomecânicos de equilíbrio e instabilidade postural com uso da plataforma de força Biodex Balance System (BBS), analisando as condições de respiração livre e respiração com um incremento na demanda inspiratória, afim de verificar influência que possa ser gerada pela maior demanda dos músculos inspiratórios na estabilidade postural de idosos.

\section{MATERIAIS E MÉTODOS}

\section{Participantes}

Foram recrutados 3 idosos do gênero masculino, participantes de programas de atividades físicas regulares, considerando os seguintes critérios de inclusão: ter idade de 60 anos ou acima e deambulante. Os critérios de exclusão foram: problema respiratório de qualquer tipo; deambular com dispositivo de auxílio (bengala, muletas, andador); presença de doença neurológica; comprometimento ortopédico nos últimos 6 meses em membros inferiores (entorses, rupturas de ligamento, lesão muscular, fraturas e cirurgias), dor lombar; comprometimento vestibular (presença de vertigem ou labirintite); comprometimento visual, não corretivo por lentes.

O estudo foi aprovado pelo comitê de ética local e todos os participantes foram informados e assinaram o termo de consentimento livre e esclarecido antes de realizar as avaliações. As coletas de dados foram realizadas no Laboratório de Biomecânica da Universidade Estadual Paulista "Julio de Mesquita Filho".

\section{Procedimientos}


Os participantes realizaram inicialmente a avaliação da pressão inspiratória máxima (PImáx) ao nível da boca com um manuvacuômetro digital Globalmed® Modelo MVD300, em seguida com uso do sistema Biodex Balance System SD (Biodex Medical Systems, Nova York, EUA), que é um instrumento projetado para medir e treinar a estabilidade postural em uma plataforma estática ou instável, foi utilizado o protocolo de software 'Postural Stability Test' (PST); e desse teste foram utilizados os dados do 'Índice de Instabilidade'. Esse sistema indica a função de equilíbrio dos indivíduos, medindo as mudanças no centro de gravidade em duas direções diferentes (anteroposterior e mediolateral) (Cho et al., 2012); um 'Índice de instabilidade' mais alto indica pior função do equilíbrio. $\mathrm{O}$ mesmo teste foi realizado em seguida com o idoso respirando através do equipamento Power Breathe ${ }^{\circledR}$, este é um dispositivo que foi programado para fazer com que a pessoa que o utiliza tenha que gerar um limiar de pressão de $50 \%$ do valor de sua PImáx, obtida na avaliação inicial; com utilização de um clipe nasal (Figura 1A-B).

Figura 1- A. Realização do PST na plataforma Biodex Balance® na condição de respiração livre. B. Realização do PST na plataforma Biodex Balance $®$ na condição de respiração com aumento na demanda inspiratória com uso do dispositivo Power Breathe ${ }$.
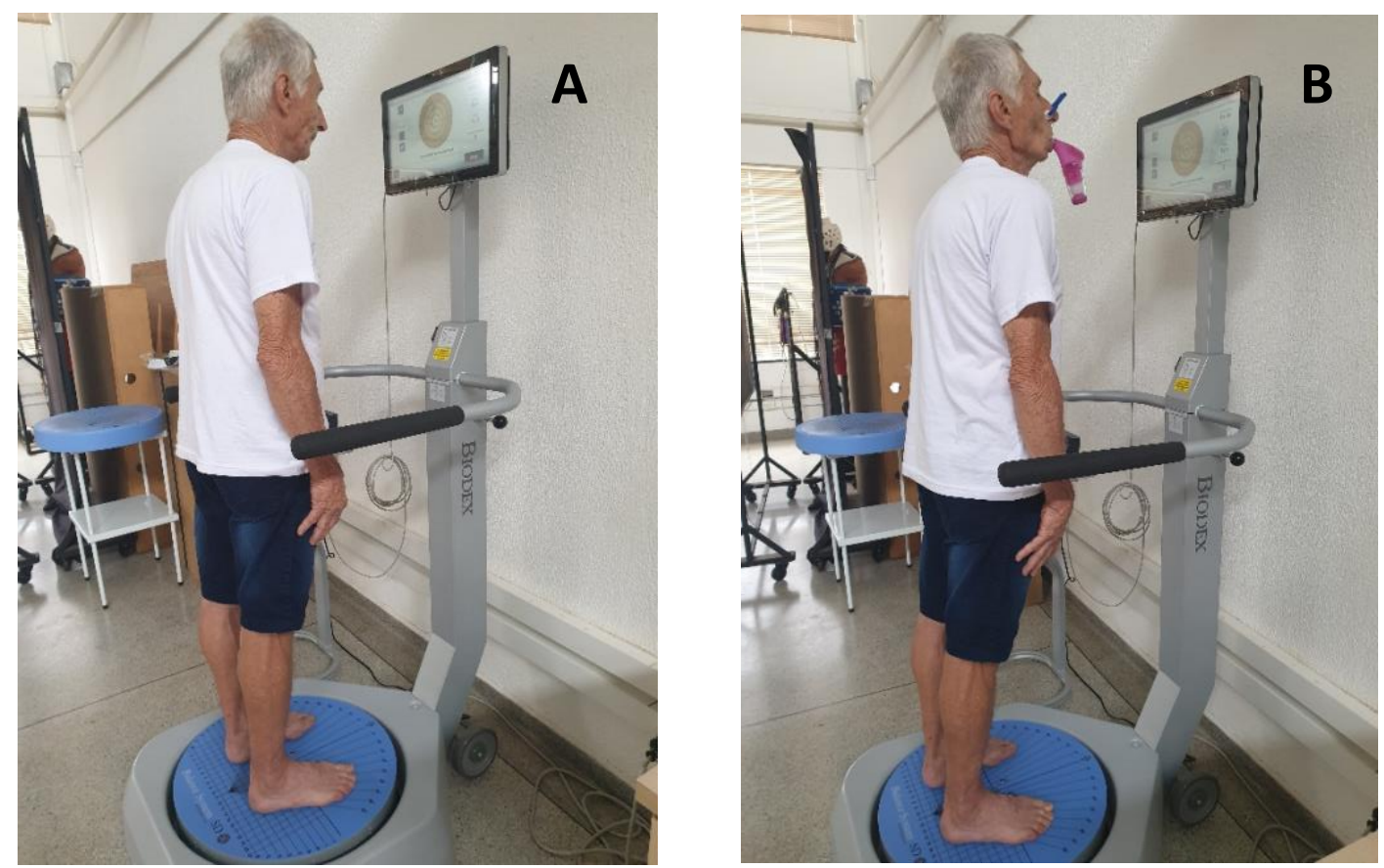

Foram investigadas as diferenças nos resultados do teste de estabilidade postural nas condições de respiração livre, e com o uso do equipamento para gerar um aumento na demanda inspiratória.

\section{RESULTADOS}

As características de cada sujeito são apresentadas na Tabela 1.

Tabela 1. Características demográficas da amostra 


\begin{tabular}{lc|c|c}
\hline & Paciente 1 & Paciente 2 & Paciente 3 \\
\hline Idade (Anos) & 79 & 70 & 77 \\
\hline Estatura (cm) & 167 & 168 & 160 \\
\hline Massa (Kg) & 65 & 51 & 81 \\
\hline IMC (Kg/cm2) & 23,31 & 18,06 & 31,64 \\
\hline
\end{tabular}

$\mathrm{Na}$ Tabela 2 são apresentados os dados do teste PST realizado na plataforma Biodex Balance ${ }^{\circledR}$ nas condições de respiração livre e com o incremento na demanda inspiratória. $\mathrm{O}$ teste avalia o índice de instabilidade postural por meio das variações nas oscilações anteriorposterior e direita-esquerda. Para as oscilações anterior-posterior todos os idosos avaliados apresentaram um maior índice de instabilidade na realização do teste com aumento da demanda inspiratória e para as oscilações direita-esquerda dois dos idosos avaliados apresentaram maior índice de instabilidade quando avaliada a condição com aumento da demanda inspiratória.

Tabela 2. Efeito de aumento na demanda inspiratória sobre a estabilidade postural de idosos.

\begin{tabular}{cc|c|c|c}
\hline \multicolumn{2}{c|}{ Índice de Instabilidade } & Paciente 1 & Paciente 2 & Paciente 3 \\
\hline Anterior/Posterior & Pré & 0,05 & 0,04 & 0,08 \\
\cline { 2 - 5 } & Sobrecarga & 0,29 & 0,31 & 0,50 \\
\hline Direita/Esquerda & Pré Deficit & $82,76 \%$ & $87,10 \%$ & $84,00 \%$ \\
\cline { 2 - 5 } & Sobrecarga & 0,05 & 0,04 & 0,14 \\
\hline & $\%$ Deficit & $80,77 \%$ & $92,59 \%$ & $0,00 \%$ \\
\hline
\end{tabular}

\section{DISCUSSÃO}

Hodges et al. (2000) já descreveram que as contrações fásicas do diafragma auxiliam na manutenção da estabilidade postural em situações em que forças externas desestabilizam a coluna. Acreditamos que um mecanismo semelhante ocorre tarefas dinâmicas e que o aumento na demanda muscular inspiratória resultou em subsequente piora nas habilidades de equilíbrio.

Acreditamos que os déficits apresentados em praticamente todas as variáveis analisadas são devido à combinação entre as alterações fisiológicas que acontecem no envelhecimento e o uso do dispositivo Power Breathe ${ }^{\circledR}$, já que existem dados de outros estudos que dizem que toda musculatura do tronco trabalha conjuntamente com a função de proteger a coluna e preservar o controle postural, e se contraem de forma automática em antecipação às ações que desestabilizam ou sobrecarregam a estabilidade postural. As contrações do diafragma ocorrem da mesma forma, mas o papel do diafragma na respiração sempre toma precedência sobre a função postural (Hodges et al., 2002). 
Apesar de esse ser apenas um estudo de casos, podemos dizer que o aumento na demanda respiratória ainda que em atividades cotidianas e atividades físicas em idosos deve ser um ponto de atenção, pois nossos dados apontam para um importante aumento no índice de instabilidade postural quando aumentada a demanda sobre os músculos inspiratórios. E sugerimos ainda que pesquisas com uma amostra maior e um estudo randomizado controlado possam ser realizados em investigações futuras para obtenção de dados mais expressivos.

\section{CONCLUSÃO}

Com esse estudo de caso concluímos que o aumento na demanda inspiratória fornecido pelo dispositivo Power Breathe ${ }^{\circledR}$ foi capaz de alterar a equilíbrio de idosos, ainda que devemos considerar que para resultados mais esclarecedores seja necessário a realização de um estudo maior, consideramos que este estudo possa ser um guia para outras pesquisas com o mesmo objetivo.

\section{Limitações do estudo}

O mecanismo exato por trás das alterações que os músculos inspiratórios podem causar no equilíbrio em idosos ainda é desconhecido, porém, pode-se dizer que tem relação com a precedência pela função fisiológica principal: a respiração. É importante considerar que este es um pequeno estúdio de casos, que avaliou três pessoas, e que para fazer suposições específicas é necessário um estudo maior. Para estudos futuros sugerimos una amostra maior em um estudo randomizado controlado.

\section{Agradecimentos:}

FAPESP Fundação de amparo à pesquisa do estado de São Paulo, na compra do equipamento Biodex Balance para o Laboratório de Biomecânica da Universidade Estadual Paulista "Júlio de Mesquita Filho"- Rio Claro-SP.

\section{REFERÊNCIAS}

Britto, R. R., Zampa, C. C., De Oliveira, T. A., Prado, L. F., \& Parreira, V. F. (2009). Effects of the aging process on respiratory function. Gerontology, 55(5), 505-510.

Caserotti, P. (2010). Strength training in older adults: changes in mechanical muscle function and functional performance. The Open Sports Sciences Journal, 3(1), 62-66.

Cho, K. H., Lee, K. J., \& Song, C. H. (2012). Virtual-reality balance training with a video-game system improves dynamic balance in chronic stroke patients. The Tohoku journal of experimental medicine, 228(1), 69-74.

Enright, P. L., Kronmal, R. A., Manolio, T. A., Schenker, M. B., \& Hyatt, R. E. (1994). Respiratory muscle strength in the elderly. Correlates and reference values. Cardiovascular Health Study Research Group. American journal of respiratory and critical care medicine, 149(2), 430-438.

Granacher, U., Gollhofer, A., Hortobágyi, T., Kressig, R. W., \& Muehlbauer, T. (2013). The importance of trunk muscle strength for balance, functional performance, and fall prevention in seniors: a systematic review. Sports medicine, 43(7), 627-641.

Hodges, P. W., Eriksson, A. M., Shirley, D., \& Gandevia, S. C. (2005). Intra-abdominal pressure increases stiffness of the lumbar spine. Journal of biomechanics, 38(9), 1873-1880.

Hodges, P. W., Gandevia, S. C. (2000). Activation of the human diaphragm during a repetitive postural task. The Journal of physiology, 522(1), 165-175.

Hodges, P. W., Gurfinkel, V. S., Brumagne, S., Smith, T. C., \& Cordo, P. C. (2002). Coexistence of stability and mobility in postural control: evidence from postural compensation for respiration. Experimental brain research, 144(3), 293-302. 
Hodges, P. W., Gurfinkel, V. S., Brumagne, S., Smith, T. C., \& Cordo, P. C. (2002). Coexistence of stability and mobility in postural control: evidence from postural compensation for respiration. Experimental Brain Research, 144(3), 293-302.

IBGE - Instituto brasileiro de geografia e estatística. Em Estimativas de Projeção da população. Projeções 1980-2050. Disponível em $\quad<\quad$ https://www.ibge.gov.br/estatisticas/sociais/populacao/9109-projecao-dapopulacao.html?=\&t=resultados $>$ el 03 de janeiro de 2020.

Janssens, L., Troosters, T., McConnell, A., Pijnenburg, M., Claeys, K., Goossens, N., ... \& Brumagne, S. (2014). Inspiratory muscle training improves proprioceptive postural control and sit-to-stand-to-sit in individuals with recurrent nonspecific low back pain. In International Society of Posture \& Gait Research congress, Date: 2014/06/292014/07/03, Location: Vancouver.

Santos, F. H. D., Andrade, V. M., \& Bueno, O. F. A. (2009). Envelhecimento: um processo multifatorial. Psicologia em estudo, 14(1), 3-10.

Studenski, S., et al. (2011). Gait speed and survival in older adults. Jama, 305(1), 50-58. 